\title{
Transferability of $\mathrm{N}$-terminal mutations of pyrrolysyl-tRNA synthetase in one species to that in another species on unnatural amino acid incorporation efficiency
}

\author{
Thomas L. Williams ${ }^{1}$ - Debra J. Iskandar ${ }^{1}$. Alexander R. Nödling ${ }^{1} \cdot$ Yurong Tan ${ }^{1}$. Louis Y. P. Luk ${ }^{1}$ Yu-Hsuan Tsai ${ }^{1}$ (I)
}

Received: 10 October 2020 / Accepted: 23 November 2020 / Published online: 17 December 2020

(c) The Author(s) 2020

\begin{abstract}
Genetic code expansion is a powerful technique for site-specific incorporation of an unnatural amino acid into a protein of interest. This technique relies on an orthogonal aminoacyl-tRNA synthetase/tRNA pair and has enabled incorporation of over 100 different unnatural amino acids into ribosomally synthesized proteins in cells. Pyrrolysyl-tRNA synthetase (PylRS) and its cognate tRNA from Methanosarcina species are arguably the most widely used orthogonal pair. Here, we investigated whether beneficial effect in unnatural amino acid incorporation caused by N-terminal mutations in PylRS of one species is transferable to PylRS of another species. It was shown that conserved mutations on the N-terminal domain of MmPylRS improved the unnatural amino acid incorporation efficiency up to five folds. As MbPylRS shares high sequence identity to MmPylRS, and the two homologs are often used interchangeably, we examined incorporation of five unnatural amino acids by four MbPylRS variants at two temperatures. Our results indicate that the beneficial N-terminal mutations in MmPylRS did not improve unnatural amino acid incorporation efficiency by MbPylRS. Knowledge from this work contributes to our understanding of PylRS homologs which are needed to improve the technique of genetic code expansion in the future.
\end{abstract}

Keywords Genetic code expansion · Unnatural amino acid · Non-canonical amino acid · Pyrrolysyl-tRNA synthetase (PylRS)

\begin{tabular}{|c|c|}
\hline \multicolumn{2}{|c|}{ Abbreviations } \\
\hline AcK & Acetyl lysine \\
\hline BocK & $\mathrm{N}^{\varepsilon}$-Boc-lysine \\
\hline IPTG & Isopropyl $\beta$-d-1-thiogalactopyranoside \\
\hline MbPylRS & $\begin{array}{l}\text { Methanosarcina barkeri Pyrrolysyl-tRNA } \\
\text { synthetase }\end{array}$ \\
\hline MmPylRS & $\begin{array}{l}\text { Methanosarcina mazei Pyrrolysyl-tRNA } \\
\text { synthetase }\end{array}$ \\
\hline PCC & Photocaged cysteine \\
\hline PylRS & Pyrrolysyl-tRNA synthetase \\
\hline
\end{tabular}

Handling editor: S. Stuchlík.

Supplementary Information The online version contains supplementary material available at https://doi.org/10.1007/s0072 6-020-02927-z.

Yu-Hsuan Tsai

tsai.y-h@outlook.com

1 School of Chemistry, Cardiff University, Cardiff CF10 3AT, UK $\begin{array}{ll}\text { PrK } & \text { Propionyl lysine } \\ \text { TAcK } & \text { Thioacetyl lysine }\end{array}$

\section{Introduction}

The ability to genetically introduce an unnatural (i.e., noncanonical) amino acid with unique chemical and physical properties into a defined position of a target protein has provided a new avenue to investigate protein function (Brown et al. 2018; Chin 2017; Nodling et al. 2019; Young and Schultz 2018). This approach known as genetic code expansion relies on an orthogonal aminoacyl-tRNA synthetase/tRNA pair to direct the site-specific incorporation of an unnatural amino acid in response to a blank codon. The amber stop codon (UAG) is usually chosen as the blank codon because it does not encode a canonical amino acid and is the least used codon in most organisms (Athey et al. 2017). Here, it is critical that the orthogonal synthetase only acylates the orthogonal tRNA with the designated unnatural amino acid, and neither the orthogonal tRNA nor the unnatural amino acid is a substrate of endogenous synthetases. 
The pyrrolysyl-tRNA synthetase (PylRS) from archaea Methanosarcina barkeri (MbPylRS) or Methanosarcina mazei (MmPylRS) and its cognate tRNA (Pyl tRNA) are arguably the most widely used orthogonal pair for genetic code expansion in bacteria and eukaryotes (Brown et al. 2018; Chin 2017; Nodling et al. 2019). The two organisms have identical sequences for Pyl tRNA (Srinivasan et al. 2002; Suzuki et al. 2017) that naturally decodes the amber codon. MbPylRS and MmPylRS also have high sequence identity (74\%). While wild-type MbPylRS and MmPylRS can recognize some unnatural amino acids, protein engineering of these homologous enzymes has enabled incorporation of over 100 different unnatural amino acids with diverse chemical and physical properties (Brown et al. 2018; Chin 2017; Nodling et al. 2019).

Despite these advances, the use of the amber codon to encode an unnatural amino acid is not without limitation. The amber codon can still be recognized by the release factor, causing translation termination and production of the truncated protein product. To circumvent this problem, different approaches have been explored. In one instance, an orthogonal ribosome has been engineered that has much lower affinity to the release factor (An and Chin 2009; Barrett and Chin 2010; Neumann et al. 2010). In fact, the orthogonal ribosome recognizes an alternative Kozak sequence, the amber codon on the reporter gene can be preferentially decoded as the unnatural amino acid, minimizing unwanted premature termination. In other approaches, the elongation factor can be engineered or the release factor can be removed to increase the amber suppression efficiency (Gan et al. 2017; Johnson et al. 2011; Schmied et al. 2014). Nevertheless, the general applicability of these approaches (An and Chin 2009; Barrett and Chin 2010; Gan et al. 2017; Johnson et al. 2011; Neumann et al. 2010; Schmied et al. 2014) is limited because the translational systems in prokaryotic cells and eukaryotic cells are vastly different and not readily interchangeable. Hence, an alternative approach that would function in both E. coli and mammalian cells is highly desirable, and this may be achieved by directly engineering the non-substrate binding part of the orthogonal synthetase to improve its catalytic activity (Owens et al. 2017; Sharma et al. 2018).

To improve unnatural amino acid incorporation, both MbPylRS and MmPylRS need to be thoroughly investigated. These homologs contain two domains, the C-terminal and $\mathrm{N}$-terminal domains. The $\mathrm{C}$-terminal domain possesses the catalytic site and binds to ATP and the unnatural amino acid. On the other hand, the N-terminal domain is important for the enzymatic activity in cells through interaction with the tRNA though is not directly involved in unnatural amino acid recognition (Suzuki et al. 2017). Often discovered through rational design, directed evolution or a combination of both, mutations in the C-terminal domain can expand the substrate scope, enabling incorporation of structurally diverse unnatural amino acids. On the other hand, though not directly involved in catalysis, mutations in the N-terminal domain have been demonstrated to affect the efficiency of unnatural amino acid incorporation in variants of MmPylRS, MbPylRS and their chimera (Bryson et al. 2017; Owens et al. 2017; Sharma et al. 2018). In a study to increase the incorporation of crotonyl lysine by a MbPylRS variant, six mutations (V8E, T13I, I36V, H45L, S121R, I355T) predominantly localized in the N-terminal domain were identified through random mutagenesis, and about threefold increase in crotonyl lysine incorporation was observed (Owens et al. 2017). When transferring these six mutations to another MbPylRS variant for incorporating $N^{\varepsilon}$-[(2-propynyloxy)carbonyl]-L-lysine, a similar beneficial effect was observed. Another study found that R19H/H29R/ T122S mutations in the N-terminal domain improved the capability of three MmPylRS variants for unnatural amino acid incorporation, and up to sixfold increase in the yields of recombinant proteins containing an unnatural amino acid was observed (Sharma et al. 2018). These studies indicate that mutations in the $\mathrm{N}$-terminal domain can affect the incorporation efficiency. However, while C-terminal mutations are transferable between MbPylRS and MmPylRS to recognize specific unnatural amino acids (Brown et al. 2018; Chin 2017; Nodling et al. 2019), it remains elusive if the $\mathrm{N}$-terminal mutations are also transferable between these two homologs.

Provided that MbPylRS and MmPylRS are often used interchangeably for unnatural amino acid incorporation (Brown et al. 2018; Chin 2017; Nodling et al. 2019), beneficial mutations found in the $\mathrm{N}$-terminal domain of one homolog are likely to be transferable to that of the other. As the MmPylRS variant R19H/H29R/T122S was reported to have improved activity, we propose that these mutations may have beneficial effect for the incorporation of unnatural amino acids by MbPylRS. As the corresponding position of MmPylRS T122 in MbPylRS is already a serine residue (Fig. 1), whereas R19 and H29 are conserved in the two

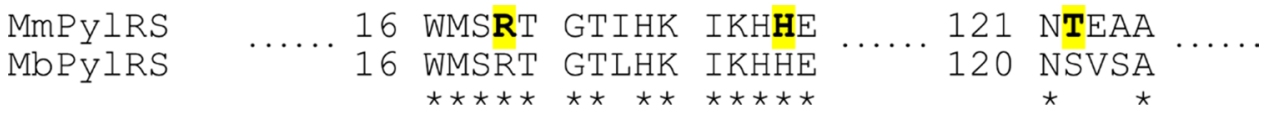

Fig. 1 Partial sequence alignment of MmPylRS and MbPylRS. Amino acid sequence of MmPylRS (UniProt: Q8PWY1) and MbPylRS (UniProt: Q6WRH6) is aligned using ExPASy (https://web.expasy.org/sim/) 
homologs. Thus, we set out to probe the effect of R19H/ $\mathrm{H} 29 \mathrm{R}$ mutations in unnatural amino acid incorporation by MbPylRS variants.

Here, we examined four MbPylRS variants (i.e., wildtype, AcKRS, PrKRS, PCCRS) for incorporating unnatural amino acids (Fig. 2) under two different temperatures $\left(37^{\circ} \mathrm{C}\right.$ or $\left.25^{\circ} \mathrm{C}\right)$. Five different unnatural amino acids $N^{\varepsilon}$ Boc-lysine (BocK), acetyl lysine (AcK), thioacetyl lysine (TAcK), propionyl lysine (PrK) and photocaged cysteine (PCC) were used here for our examination. BocK can be incorporated by wild-type MbPylRS and is often used as a model unnatural amino acid in proof-of-principle studies. Both AcK and PrK are commonly used to investigate the effect of lysine post-translational modifications (i.e., lysine acetylation and propionylation, respectively) in bacteria and eukaryotic cells (Drazic et al. 2016; Ju and He 2017; Lin et al. 2012). TAcK is a stable analog mimicking lysine acetylation, but the carbonyl functionality is replaced with a thiocarbonyl group thereby preventing enzymatic hydrolysis (Venkat et al. 2017; Xiong et al. 2016). Due to the structural similarity, AcK and PrK can be incorporated by both AcKRS and PrKRS, and TAcK is likely to be accepted by these PylRS variants as well. On the other hand, PCC has a distinct structure when compared to the lysine analogs. PCC has a photolabile protecting group on the cysteine side chain, useful in light-controlled protein activation of active cysteines (Nguyen et al. 2014).

Overall, we found that R19H/H29R mutations have negligible benefits to MbPylRS variants. In two cases (i.e., PrKRS and PCCRS), the N-terminal mutations greatly decreased the unnatural amino acid incorporation. Our results highlight the difference between MbPylRS and MmPylRS, two closely related homologs, and raise concerns of using these homologs interchangeably in genetic code expansion.

\section{Methods}

\section{Unnatural amino acids}

BocK (Fluorochem, \#078,520) and AcK (Fluorochem, $\# 324,288$ ) are commercially available. TAcK (Venkat et al. 2017), PrK (Gattner et al. 2013) and PCC (Nguyen et al. 2014) were synthesized following the literature procedures.

\section{Cloning}

A MbPylRS variant and the Pyl tRNA are expressed from a pCDF vector, in which the synthetase and the tRNA are under the control of constitutively active GlnS promoter and Ipp promoter, respectively. Four variants of MbPylRS were tested here, including the wild-type enzyme, MbAcKRS (D76G/S123G/L266M/L270I/Y271F/L274A/C313F) (Neumann et al. 2009), MbPrKRS (Y271F/C313T) (Wilkins et al. 2015) and MbPCCRS (N311Q/C313A/V366M) (Nguyen et al. 2014). Apart from the wild-type enzyme, the other variants contain mutations indicated in the brackets for recognizing the designated unnatural amino acid (Table 1).

Plasmids PylST (Neumann et al. 2010), AcKST (Neumann et al. 2009) and PCCST (Nguyen et al. 2014) are kind gifts from Jason Chin. Plasmid PrKST was constructed by two rounds of PCR. Firstly, Y271F mutation was introduced using PylST as the template and the primers AACTATCTG CGTAAACTGGATCGTATTCTG and TCCAGTTTACGC AGATAGTTAAACAGGGTCGGGGCCAGCATC for PCR. The resulting PCR product $(6 \mathrm{~kb})$ was directly transformed into E. coli Stbl3 (ThermoFisher, \#C737303), and plasmids were extracted from cells surviving in spectinomycin $(50 \mu \mathrm{g} /$ $\mathrm{ml}$ ) containing media. The extracted plasmid served as the
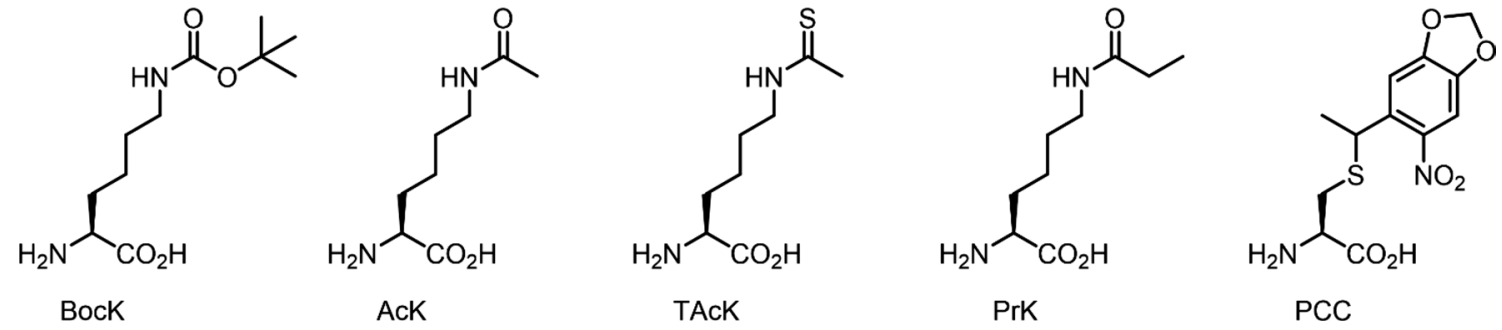

Fig. 2 Structures of the five unnatural amino acids used in this study

Table 1 Comparison of C-terminal mutations in the four MbPylRS variants used in this study

\begin{tabular}{llllllllll}
\hline Residue no. & 76 & 123 & 266 & 270 & 271 & 274 & 311 & 313 & 366 \\
\hline MbPylRS & D & S & L & L & Y & L & N & C & V \\
MbAcKRS & G & G & M & I & F & A & N & F & V \\
MbPrKRS & D & S & L & L & F & L & N & T & V \\
MbPCCRS & D & S & L & L & Y & L & Q & A & M \\
\hline
\end{tabular}


template for the second round of PCR with primers AAT TCACCATGGTTAACTTTACCCAAATGGGCAGCG GCTGCAC and AAAGTTAACCATGGTGAATTCTTC CAGGTG to introduce mutation $\mathrm{C} 313 \mathrm{~T}$, providing plasmid PrKST.

The N-terminal mutations (R19H/H29R) were introduced into each PylRS variant by PCR using primers TGGATG AGCCATACCGGCACCCTGCATAAAATCAAACATCGT GAAGTGAG and TGCCGGTATGGCTCATCCACAGGC CGGTCGCGCTAATCAGCACATC. All PCR reactions were performed using PrimeStar Max DNA polymerase (TaKaRa, \#R045A).

Plasmid pET sfGFP(150TAG) has been deposited at the Addgene (Plasmid \#133,455) and was constructed in two steps. Plasmid pBAD sfGFP (Reddington et al. 2012), a kind gift from Dafydd Jones, was digested with restriction enzyme NcoI and XhoI to excise the gene encoding sfGFP, which was cloned into a pET 28a vector using the same restriction sites to afford pET sfGFP. The mutation N150TAG was introduced by PCR using primers AATATA AATTCAACAGCCATTAGGTGTATATTACCGATAAAC AG and TGGCTGTTGAAATTATATTCCAGTTTATGA CC.

\section{Determining unnatural amino acid incorporation efficiency}

The workflow is illustrated in Fig. 3. Specifically, chemically competent $E$. coli BL21(DE3) cells were transformed with the reporter plasmid, pET sfGFP(150TAG), and the appropriate PylRS plasmid. The transformation was conducted by incubation with plasmids on ice for $10 \mathrm{~min}$, heat shock at $42{ }^{\circ} \mathrm{C}$ for $45 \mathrm{~s}$, incubation on ice for $2 \mathrm{~min}$, and recovery in fresh $\mathrm{LB}$ media at $37{ }^{\circ} \mathrm{C}$ for $1 \mathrm{~h}$ with constant agitation. Cells were added into fresh LB media $(10 \mathrm{ml})$ containing kanamycin $(50 \mu \mathrm{g} / \mathrm{ml}$, selection marker of the reporter plasmid) and spectinomycin $(50 \mu \mathrm{g} / \mathrm{ml}$, selection marker of the PylRS plasmid). Cells were cultured at $37^{\circ} \mathrm{C}$ overnight with constant agitation.

The overnight culture was diluted into fresh LB media $(25 \mathrm{ml})$ containing kanamycin $(50 \mu \mathrm{g} / \mathrm{ml})$ and spectinomycin $(50 \mu \mathrm{g} / \mathrm{ml})$ to reach $\mathrm{OD}_{600}=0.1$. This culture was incubated at $37{ }^{\circ} \mathrm{C}$ with constant agitation until $\mathrm{OD}_{600}=0.6-0.8$, and isopropyl $\beta-\mathrm{D}-1$ thiogalactopyranoside (IPTG) was added to reach the final concentration of $1 \mathrm{mM}$. The culture was split into $50-\mathrm{ml}$ falcon tubes (5-ml culture per tube), and unnatural amino acid was added, if required, to reach the final concentration of $5 \mathrm{mM}$ (except for BocK, final concentration $=1 \mathrm{mM}$ ). Cultures were incubated at $25^{\circ} \mathrm{C}$ or $37{ }^{\circ} \mathrm{C}$ for $18 \mathrm{~h}$ with constant agitation for protein production.

After incubation, $\mathrm{OD}_{600}$ of the cultures were recorded, and samples $(1 \mathrm{ml})$ were taken from each culture. The samples were centrifuged at $20{ }^{\circ} \mathrm{C}$ and $21,130 \times g$ for $5 \mathrm{~min}$. Supernatants were removed, and the pellets were resuspended in PBS. The samples were centrifuged again at $20^{\circ} \mathrm{C}$ and $21,130 \times g$ for $5 \mathrm{~min}$. Supernatants were removed, and the pellets were resuspended in PBS to $\mathrm{OD}_{600}=1.0$. The resuspension $(200 \mu \mathrm{l})$ was placed into a 96-well plate, and the fluorescence was measured by a BMG Labtech FLUOstar OPTIMA microplate reader (excitation wavelength $=485 \mathrm{~nm}$, emission wavelength $=520 \mathrm{~nm}, 20$ flashes per well, 0.2 position delay, gain $=1000$, shaking for $30 \mathrm{~s}$ before plate reading at $25{ }^{\circ} \mathrm{C}$ ). Three biological repeats of each expression condition and two technical repeats of each fluorescent measurement were performed.
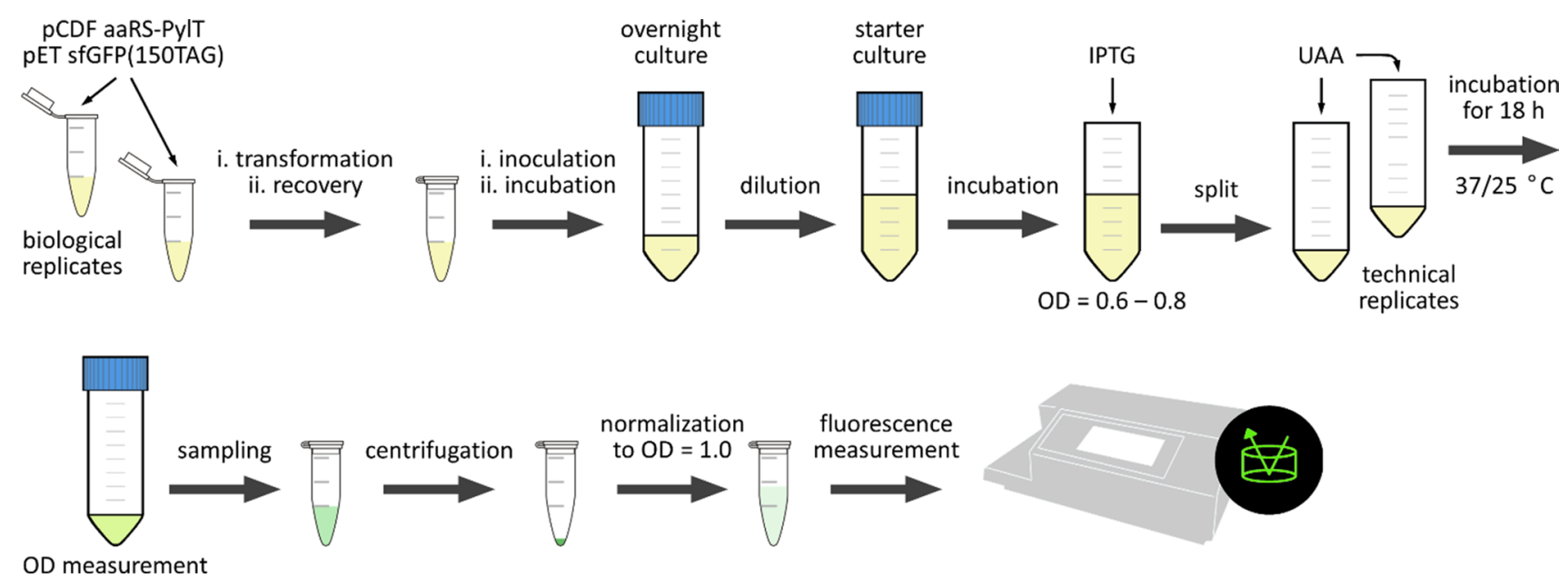

Fig. 3 Experimental workflow. Cells were incubated at $37{ }^{\circ} \mathrm{C}$ unless otherwise stated. Biological replicates were prepared from independent transformations. Technical replicates were prepared from the IPTG-induced cultures. See text description for details 

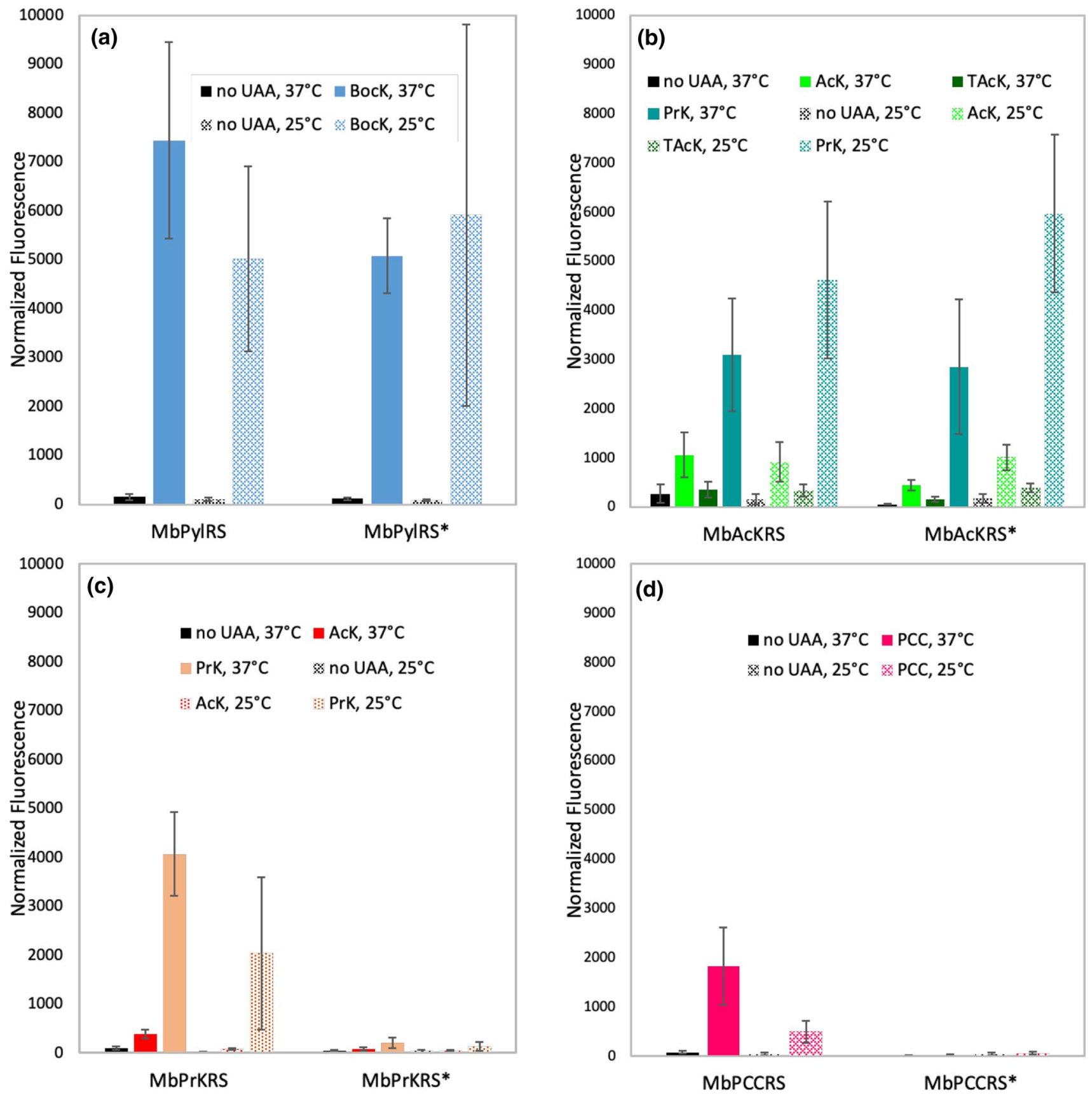

Fig. 4 Unnatural amino acid incorporation efficiency by different MbPylRS variants. Transformed E. coli BL21(DE3) were normalized to $\mathrm{OD}_{600}=1.0$ before fluorescence measurement. Means and standard deviations are plotted, and their values are listed in Table 2.

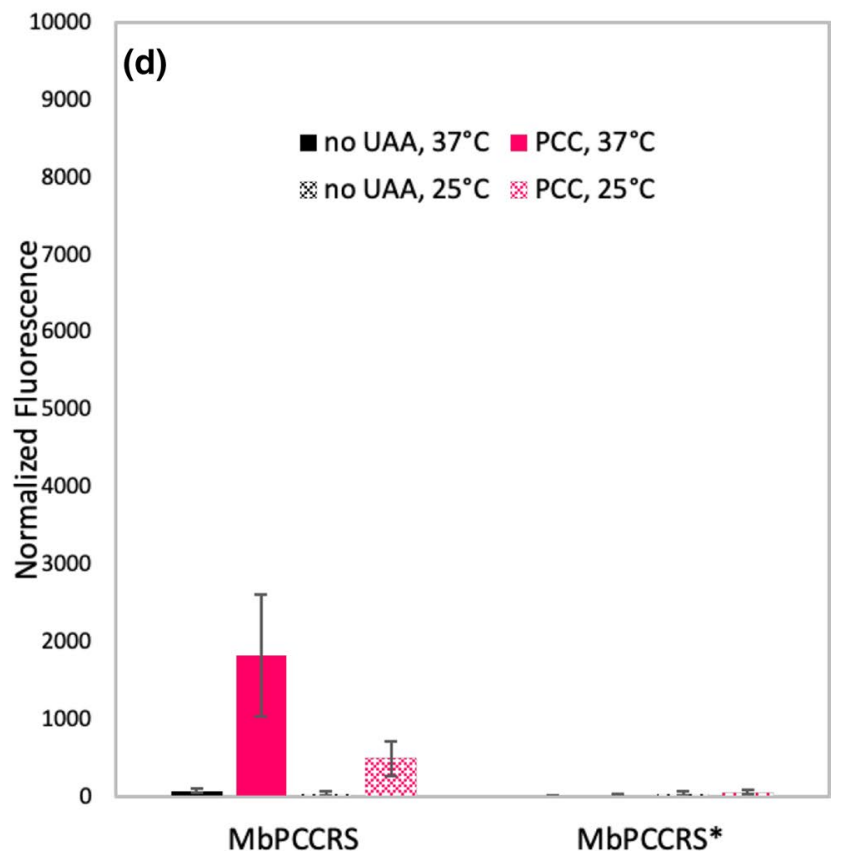

The asterisk (*) denotes synthetase variants contain additional R19H/ H29R mutations in the N-terminal domain. UAA: unnatural amino acid. Fluorescence analysis of a wild-type MbPylRS, b AcKRS, c PrKRS and $\mathbf{d}$ PCCRS

\section{Results}

Our aim is to examine if the beneficial effect of R19H/ H29R mutations found in the study of MmPylRS (Sharma

et al. 2018) is transferable to MbPylRS. To investigate, we employed a fluorescence-based assay. Synthetase variants containing additional R19H/H29R mutations in the $\mathrm{N}$-terminal domain of PylRS are denoted with an asterisk (*). For example, MbPylRS refers to the synthetase with 
Table $2 P$ values of paired $t$ tests comparing the effect of R19H/H29R in MbPylRS variants

\begin{tabular}{|c|c|c|c|c|c|}
\hline \multirow[t]{2}{*}{ Entry } & \multirow[t]{2}{*}{ Temperature } & \multirow[t]{2}{*}{ UAA } & \multicolumn{2}{|c|}{ Normalized fluorescence (mean \pm standard deviation) } & \multirow[t]{2}{*}{$P$ value } \\
\hline & & & Wild-type N-terminal & $\mathrm{R} 19 \mathrm{H} / \mathrm{H} 29 \mathrm{R}$ & \\
\hline 1 & $37^{\circ} \mathrm{C}$ & BocK & MbPylRS $(7443 \pm 2010)$ & MbPylRS* $(5081 \pm 764)$ & $1.0 \times 10^{-2}$ \\
\hline 2 & $25^{\circ} \mathrm{C}$ & BocK & MbPylRS $(5023 \pm 1895)$ & MbPylRS* $(5919 \pm 3900)$ & $5.4 \times 10^{-1}$ \\
\hline 3 & $37^{\circ} \mathrm{C}$ & AcK & $\operatorname{MbAcKRS}(1059 \pm 459)$ & MbAcKRS* $(443 \pm 105)$ & $2.9 \times 10^{-3}$ \\
\hline 4 & $25^{\circ} \mathrm{C}$ & AcK & MbAcKRS $(912 \pm 403)$ & MbAcKRS* $(1013 \pm 255)$ & $6.1 \times 10^{-1}$ \\
\hline 5 & $37^{\circ} \mathrm{C}$ & TAcK & $\operatorname{MbAcKRS}(360 \pm 165)$ & $\operatorname{MbAcKRS*}(157 \pm 51)$ & $6.1 \times 10^{-3}$ \\
\hline 6 & $25^{\circ} \mathrm{C}$ & TAcK & $\operatorname{MbAcKRS}(339 \pm 129)$ & $\operatorname{MbAcKRS*}(390 \pm 83)$ & $3.9 \times 10^{-1}$ \\
\hline 7 & $37^{\circ} \mathrm{C}$ & PrK & $\operatorname{MbAcKRS}(3104 \pm 1147)$ & MbAcKRS* $(2851 \pm 1375)$ & $7.4 \times 10^{-1}$ \\
\hline 8 & $25^{\circ} \mathrm{C}$ & PrK & $\operatorname{MbAcKRS}(4621 \pm 1590)$ & MbAcKRS* $(5972 \pm 1610)$ & $1.4 \times 10^{-1}$ \\
\hline 9 & $37^{\circ} \mathrm{C}$ & AcK & MbPrKRS $(384 \pm 81)$ & $\operatorname{MbPrKRS} *(85 \pm 38)$ & $8.3 \times 10^{-7}$ \\
\hline 10 & $25^{\circ} \mathrm{C}$ & AcK & MbPrKRS $(77 \pm 25)$ & MbPrKRS* $(42 \pm 13)$ & $1.3 \times 10^{-2}$ \\
\hline 11 & $37^{\circ} \mathrm{C}$ & PrK & $\operatorname{MbPrKRS}(4067 \pm 853)$ & MbPrKRS* $(203 \pm 114)$ & $1.2 \times 10^{-7}$ \\
\hline 12 & $25^{\circ} \mathrm{C}$ & PrK & $\operatorname{MbPrKRS}(2039 \pm 1556)$ & MbPrKRS* $(135 \pm 88)$ & $1.4 \times 10^{-2}$ \\
\hline 13 & $37^{\circ} \mathrm{C}$ & PCC & MbPCCRS (1828 \pm 795$)$ & MbPCCRS* $(21 \pm 8)$ & $1.2 \times 10^{-6}$ \\
\hline 14 & $25^{\circ} \mathrm{C}$ & PCC & MbPCCRS $(494 \pm 229)$ & MbPCCRS* $(60 \pm 26)$ & $1.0 \times 10^{-5}$ \\
\hline
\end{tabular}

$P$ values $<0.05$ are shown in bold. Original fluorescence data are in S2 data the wild-type sequence, whereas MbPylRS* refers to the synthetase containing R19H/H29R mutations with respect to the wild-type sequence. In our assay, production of fulllength and functional sfGFP only takes place when the amber codon is successfully suppressed (i.e., decoded by the Pyl tRNA for unnatural amino acid incorporation). As the fluorescence intensity is proportional to the quantity of fulllength, functional sfGFP, it provides a quantitative measure with regards to the unnatural amino acid incorporation.

In all MbPylRS synthetase variants tested, R19H/H29R mutations did not improve the incorporation efficiency over the wild-type sequence for any of the five amino acids (Fig. 4). At $25^{\circ} \mathrm{C}, \mathrm{R} 19 \mathrm{H} / \mathrm{H} 29 \mathrm{R}$ mutations seemed to be beneficial to PylRS (Fig. 4a) and AcKRS (Fig. 4b) at the first glance. However, upon statistical analysis, the mean normalized fluorescence values for BocK incorporation by MbPylRS at $25^{\circ} \mathrm{C}$ (Table 2, entry 2), as well as for AcK, TAcK or PrK incorporation by MbAcKRS at $25^{\circ} \mathrm{C}$ (Table 2, entries $4,6,8$ ) are not statistically significant different $(P>0.1)$ between the variants with the wild-type and the N-terminal mutations. Intriguingly, the N-terminal mutations greatly diminished the activity of PrKRS (Fig. 4c) and PCCRS (Fig. 4d). On the other hand, it is noteworthy that PrK is a better substrate than AcK in both PrKRS and AcKRS.

\section{Discussion}

Here, we investigated the effect of R19H/H29R mutations in MbPylRS variants for unnatural amino acid incorporation. We tested four MbPylRS variants (i.e., wild-type,
AcKRS, PrKRS, PCCRS), five unnatural amino acids (BocK, AcK, TAcK, PrK, PCC), and two culture temperatures $\left(37{ }^{\circ} \mathrm{C}\right.$ or $\left.25^{\circ} \mathrm{C}\right)$. While for three MmPylRS variants it was found that R19H/H29R mutations together with T122S mutation (corresponding residue of MmPyl Thr122 is already Ser, Fig. 1) improved unnatural amino acid incorporation (12), MbPylRS variants containing R19H/H29R mutations do not show any beneficial effect.

There is no correlation between point mutations in the C-terminus of the PylRS variants and the effect of $\mathrm{N}$-terminal mutations on incorporation efficiency. This is evident by the lack of activity observed with MbPrKRS* and MbPCCRS*, negligible difference was observed between MbPylRS and MbPylRS*, as well as MbAcKRS and MbAcKRS*. It is likely that the two closely related homologs, while often used interchangeably in genetic code expansion (Brown et al. 2018; Chin 2017; Nodling et al. 2019), are different in their underlying molecular mechanisms for functional regulation and catalytic activity. Indeed, there are 35 extra amino acid residues in the N-terminal domain of MmPylRS, and this difference accounts for the difference in the length of the two homologs (MmPylRS: 454 amino acids; MbPylRS: 419 amino acids). On the other hand, wild-type MmPylRS and MbPylRS have been reported with different substrate scope. For example, while $N^{\varepsilon}$-L-thiaprolyl-L-lysine and $N^{\varepsilon}$-L-cysteinyl-L-lysine are barely recognized by wildtype MmPylRS as a substrate, they can be readily incorporated into a protein using wild-type MbPylRS (Nguyen et al. 2011). To improve unnatural amino acid incorporation efficiency, it would be necessary to perform detailed 
investigation of each homolog independently and caution should be taken when transferring mutations between MmPylRS and MbPylRS.

\section{Limitations}

We have only tested the transferability of one set of N-terminal mutations between MmPylRS and MbPylRS.

Acknowledgements We are grateful to Cardiff University for the financial support.

Author contributions TLW: conceptualization. DJI, TLW, ARN, YT: data curation. LYPL, YHT: formal analysis. LYPL, YHT: funding acquisition. DJI, TLW, ARN, YT: investigation. TLW, ARN, YHT: methodology. LYPL, YHT: project administration. LYPL, YHT: resources. LYPL, YHT: supervision. YHT: validation. YHT: visualization. LYPL, YHT: writing-original draft preparation. TLW, DJI, ARN, YT, LYPL, YHT: writing-review and editing.

Funding This project was supported by Cardiff University. The funders had no role in study design, data collection and analysis, decision to publish, or preparation of the manuscript.

Data availability Data are available in the two supplementary files.

\section{Compliance with ethical standards}

Conflict of interest The authors have declared that no competing interests exist.

\section{Ethics approval and consent to participate Not applicable.}

Consent for publication The authors declare that there is no duality of interest associated with this manuscript, and all the authors consent to publish this manuscript.

Open Access This article is licensed under a Creative Commons Attribution 4.0 International License, which permits use, sharing, adaptation, distribution and reproduction in any medium or format, as long as you give appropriate credit to the original author(s) and the source, provide a link to the Creative Commons licence, and indicate if changes were made. The images or other third party material in this article are included in the article's Creative Commons licence, unless indicated otherwise in a credit line to the material. If material is not included in the article's Creative Commons licence and your intended use is not permitted by statutory regulation or exceeds the permitted use, you will need to obtain permission directly from the copyright holder. To view a copy of this licence, visit http://creativecommons.org/licenses/by/4.0/.

\section{References}

An WL, Chin JW (2009) Synthesis of orthogonal transcriptiontranslation networks. Proc Natl Acad Sci USA 106:8477-8482

Athey J, Alexaki A, Osipova E, Rostovtsev A, Santana-Quintero LV, Katneni U, Simonyan V, Kimchi-Sarfaty C (2017) A new and updated resource for codon usage tables. BMC Bioinform 18:391

Barrett OPT, Chin JW (2010) Evolved orthogonal ribosome purification for in vitro characterization. Nucleic Acids Res 38:2682-2691

Brown W, Liu JH, Deiters A (2018) Genetic code expansion in animals. ACS Chem Biol 13:2375-2386

Bryson DI, Fan C, Guo LT, Miller C, Soll D, Liu DR (2017) Continuous directed evolution of aminoacyl-tRNA synthetases. Nat Chem Biol 13:1253-1260

Chin JW (2017) Expanding and reprogramming the genetic code. Nature 550:53-60

Drazic A, Myklebust LM, Ree R, Arnesen T (2016) The world of protein acetylation. Biochim Biophys Acta 1864:1372-1401

Gan R, Perez JG, Carlson ED, Ntai I, Isaacs FJ, Kelleher NL, Jewett MC (2017) Translation system engineering in Escherichia coli enhances non-canonical amino acid incorporation into proteins. Biotechnol Bioeng 114:1074-1086

Gattner MJ, Vrabel M, Carell T (2013) Synthesis of epsilon-N-propionyl-, epsilon-N-butyryl-, and epsilon-N-crotonyl-lysine containing histone $\mathrm{H} 3$ using the pyrrolysine system. Chem Commun 49:379-381

Johnson DBF, Xu JF, Shen ZX, Takimoto JK, Schultz MD, Schmitz RJ, Xiang Z, Ecker JR, Briggs SP, Wang L (2011) RF1 knockout allows ribosomal incorporation of unnatural amino acids at multiple sites. Nat Chem Biol 7:779-786

Ju Z, He JJ (2017) Prediction of lysine propionylation sites using biased SVM and incorporating four different sequence features into Chou's PseAAC. J Mol Graphics Model 76:356-363

Lin H, Su X, He B (2012) Protein lysine acylation and cysteine succination by intermediates of energy metabolism. ACS Chem Biol 7:947-960

Neumann H, Hancock SM, Buning R, Routh A, Chapman L, Somers J, Owen-Hughes T, van Noort J, Rhodes D, Chin JW (2009) A method for genetically installing site-specific acetylation in recombinant histones defines the effects of H3 K56 acetylation. Mol Cell 36:153-163

Neumann H, Wang KH, Davis L, Garcia-Alai M, Chin JW (2010) Encoding multiple unnatural amino acids via evolution of a quadruplet-decoding ribosome. Nature 464:441-444

Nguyen DP, Elliott T, Holt M, Muir TW, Chin JW (2011) Genetically encoded 1,2-aminothiols facilitate rapid and site-specific protein labeling via a bio-orthogonal cyanobenzothiazole condensation. J Am Chem Soc 133:11418-11421

Nguyen DP, Mahesh M, Elsasser SJ, Hancock SM, Uttamapinant C, Chin JW (2014) Genetic encoding of photocaged cysteine allows photoactivation of TEV protease in live mammalian cells. J Am Chem Soc 136:2240-2243

Nodling AR, Spear LA, Williams TL, Luk LYP, Tsai Y-H (2019) Using genetically incorporated unnatural amino acids to control protein functions in mammalian cells. Essays Biochem 63:237-266

Owens AE, Grasso KT, Ziegler CA, Fasan R (2017) Two-tier screening platform for directed evolution of aminoacyl-tRNA synthetases with enhanced stop codon suppression efficiency. ChemBioChem 18:1109-1116

Reddington SC, Tippmann EM, Jones DD (2012) Residue choice defines efficiency and influence of bioorthogonal protein modification via genetically encoded strain promoted Click chemistry. Chem Commun 48:8419-8421

Schmied WH, Elsasser SJ, Uttamapinant C, Chin JW (2014) Efficient multisite unnatural amino acid incorporation in mammalian cells via optimized pyrrolysyl tRNA synthetase/tRNA expression and engineered eRF1. J Am Chem Soc 136:15577-15583

Sharma V, Zeng Y, Wang WW, Qiao Y, Kurra Y, Liu WR (2018) Evolving the $\mathrm{N}$-terminal domain of pyrrolysyl-tRNA synthetase 
for improved incorporation of noncanonical amino acids. ChemBioChem 19:26-30

Srinivasan G, James CM, Krzycki JA (2002) Pyrrolysine encoded by UAG in Archaea: charging of a UAG-decoding specialized tRNA. Science 296:1459-1462

Suzuki T, Miller C, Guo LT, Ho JML, Bryson DI, Wang YS, Liu DR, Soll D (2017) Crystal structures reveal an elusive functional domain of pyrrolysyl-tRNA synthetase. Nat Chem Biol 13:1261-1266

Venkat S, Nannapaneni DT, Gregory C, Gan Q, McIntosh M, Fan C (2017) Genetically encoding thioacetyl-lysine as a non-deacetylatable analog of lysine acetylation in Escherichia coli. FEBS Open Bio 7:1805-1814

Wilkins BJ, Hahn LE, Heitmuller S, Frauendorf H, Valerius O, Braus GH, Neumann H (2015) Genetically encoding lysine modifications on histone H4. ACS Chem Biol 10:939-944
Xiong H, Reynolds NM, Fan C, Englert M, Hoyer D, Miller SJ, Soll D (2016) Dual genetic encoding of acetyl-lysine and non-deacetylatable thioacetyl-lysine mediated by flexizyme. Angew Chem Int Ed 55:4083-4086

Young DD, Schultz PG (2018) Playing with the molecules of life. ACS Chem Biol 13:854-870

Publisher's Note Springer Nature remains neutral with regard to jurisdictional claims in published maps and institutional affiliations. 